Die Schrift weist tuf die erste Hälfte des vierten Jahrb. Zu derselben Familie gehört der auf einer Basis aus dem vierten Jahrh. (ebenfills in Rhamnus gefunden)

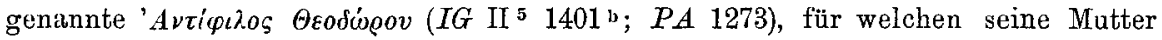

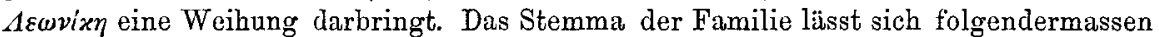
autstellen:

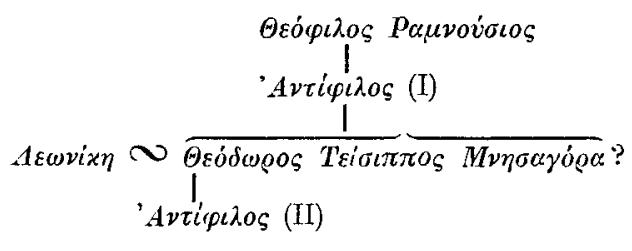

\title{
Die Festsetzung der Grenze im Frieden des Antiochus.
} Von Panl Viereck.

Mommsen hat in den Römischen Forschungen Bd. II S. 510 ff. die Ueberlieferung über den Frieden mit Antiochus einer genauen Untersuchung unterzogen, in deren Verlauf er über die Festsetzung der Grenze zu einem Resultat gekonmen ist, das, wie sich mir schon vor vielen Jahren beim Studium des Aufsatzes ergab, nicht richtig sein kann. Die ausführliche Begründung meiner abweichenden Ansicht und einer damit verbundenen Textänderung bei livius sollen die folgenden Zeilen geben ${ }^{\mathfrak{}}$ ).

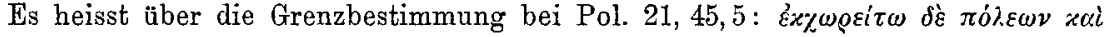

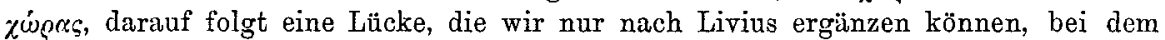
es in der Uebersetzung des Polybianischen Textes $(38,38,4)$ heisst: excedito urbibus agris vicis castellis cis Taurum montem usque ad Tanaim (einige jüngere Handschriften ad accaym oder accayn oder ad canym oder acchanim) amnem et ea (a Paris.) valle Tauri usque ad iuga (ab inga Bamb., die andern ad iugum) qua in Lycaoniam (lycaonia Bamb.) vergit. Dass der Name des Grenzflusses Tanaim oder Accayn u. s. w. verdorben ist, scheint zweifellos und ist auch von allen anerkannt; denn ein solcher Fluss ist in Kleinasien unbekannt. Da nun Strabo ${ }^{2}$ ) das Resultat der Kämpfe der Römer in 6. Jahrh. der Stadt für Asien dahin zusammenfasst, dass der Teil Kleinasiens, der innerhalb des Halys und Taurus liege, den Römern untertan geworden sei, so hat man statt Tanaim us que ad $\mathrm{H}$ a l y n a m n e m eingesetzt.

Hiergegen erbebt Mommsen Einspruch. Erstens, meint er, habe diese Bestimmung in den Vertrag nicht aufgenommen werden können, da sich des Königs Machtgebiet auf die Süd- und Westküste beschränkt habe, Kappadokien, Galatien, Bithynien garnicht in seiner Gewalt gewesen seien, die Galater vielmehr - wir können gleich hinzusetzen, auch die Lykaonier und Kappadokier - als selbständige Bunclesgenossen mit Antiochus gegen die Römer gekämpft hätten. $\mathrm{Z}$ we ite ns wendet er ein, das Taurusgebirge als Grenze zu setzen ohne nähere Definition, sei eine Unvernünftigkeit, die in einem Vertrage nicht habe stehen können, drittens vermutet er, der als Grenzscheide angegebene Fluss müsse ein von clen Höhen des Taurus kommender Fluss sein, und das sei der von Livius 38, 15, 7 bei

1) Direktor Müller, dem ich kürzlich meine Ansicht mitteilte, hat sich ihr in der Weissenbornschen Liviusausgabe (Sammlung Haupt-Sauppe, Bd. 8, Heft 2, Buch XXXVII-XXXVIII 3. Aufl. Weidmann 1907) in seinem Kommentar angeschlossen und auch die Textänderung aufgenommen (vgl. die krit. Anm. zu 38, 38, 4 S. 292).

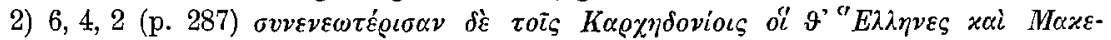

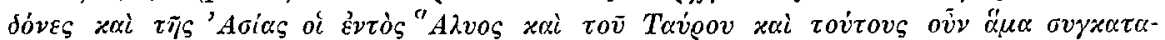

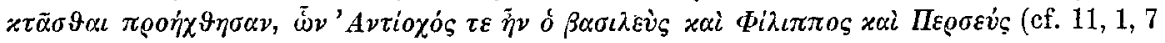
p. 492). 
der Erzählung von dem Rückmarsch des Volso aus Pamphylien erwiihnte Taurusfluss ${ }^{1}$ ) - Tanaim sei also aus Tiuurum verdorben - und als Tiurus sei von Livius der Kestros bezeichnet worden "), der Pamphylien bekanntlich in eine westliche und östliche Hülfte teilt. Diese Vermutung sucht er durch zwei Dingé zu stützen. Erstens berichtet Pol. 21,48, 11 im Anschluss an die definitiven von Volso und der

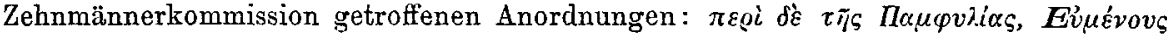

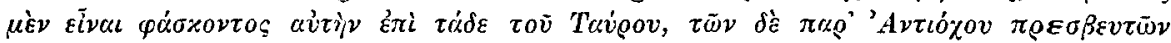

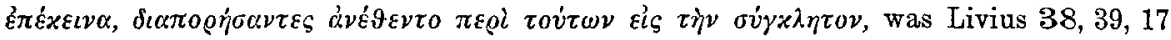
übersetzt: de Pamphylia disceptatum inter Eumenem et Antiochi legatos cum csset, quia pars eius citra, pars ultra Taurum est, integra res ad senatum reicitur. Diese Stellen, meint Mommsen, bewiesen, dass sich gleich nach dem Abschluss des Friedens ein Streit über die durch die Worte usque ad Taurum amnem bezeichnete Grenzlinie erhoben habe, weil eben dieser Grenzfluss seinem Namen nach nicht zu identifizieren gewesen wäre. Sodann glaubt Mommsen, dass die Grenze in Pamphylien zu suchen sei, ergebe sich aus Pol. 21, 35, 4 f. (= Liv. 38, 15, 6 f.) und 21, 44, 1 ff. (= Liv. 38, 37, 9 f.). An der ersten Stelle wird erzählt, dass Volso bis Termessos marschiert sei und Gesandtschaften aus den Stïdten Pamphyliens, übrigens auch aus Aspendos östlich vom Kestros, empfangen und mit Termessos und Aspendos Freundschaftsvertrïge geschlossen habe, an der zweiten, dass der Kommandant von Perge, das westlich vom Kestros lag, auf Verlangen Volsos, nach Einholung der Zustimmung seines Königs Antiochus, die Stadt gerüumt habe, und die hat er eben nach Mommsen rïumen müssen, weil das Land westlich vom Tauros-Kestros von Antiochus an die Römer durch den Friedensvertrag abgetreten worden sei.

Dieser ganzen Beweisführung kann ich nicht zustimmen, und ich will in umgekehrter Reihenfolge, mit dem letzten beginnend, sie zu widerlegen versuchen. Aus dem Zuge des Volso nach Pamphylien und aus seinen Unterhandlungen mit dem Kommandanten von Perge lässt sich absolut nicht darauf schliessen, dass der Kestros als Grenze festgesetzi war. Denn als Volso nach Termessos heranrückt, empfüngt er die Gesandtschaften von allen Städten Pamphyliens, schliesst auch mit den Aspendiern, die in dem von Mommsen Antiochus zugesprochenen Teil Pamphyliens wohnen, einen Freundschaftsvertrag ${ }^{3}$ ). Als er zum zweiten Male heranzieht, ist sein Zweck, den Kommandanten von Perge zur Rüumung der Stadt zu veranlassen, was er ja auch erreicht. Ob ïberhaupt noch andere Truppen des Antiochus in Pamphy. lien standen, wird mit keiner Silbe angedeutet. Eher könnte man aus der Art, wie Polybius dies erzïhit, schliessen, dass allein in Perge ein pflichttrever Kommandant des Antiochus zurückgeblieben sei. Diss er deswegen Perge habe räumen müssen, weil es auf dem linken Ufer des Kestros gelegen und dieser Teil Pamphyliens von Antiochus abgetreten worden sei, dessen geschieht mit keinem Worte Erwïhnung. Es kommt hinzu, dass die Römer, da sie sonst immer über die ganzen, ungeteilten Landschaften Kleinasiens Bestimmungen treffen, ob sie in Abhïngigkeit von Antiochus stehen sollen oder nicht, das kleine Pamphylien schwerlich zwischen sich und Antiochus geteilt haben werden. Es ist auch nicht der geringste Grund dafür ausfindig zu machen. Dass aber diese meine Betracbtungen richtig sind, wird sicher bestätigt durch die

1) Ex Pamphylia rediens ad fuvium Taurum primo die, postero ad Xylinen quam vocant Comen (unbekannt) posuit castra.

2) Dies scheint nach dem Zusammenhang, wenn nicht sicher, doch möglich oder, wenn man will, wahrscheinlich zu sein.

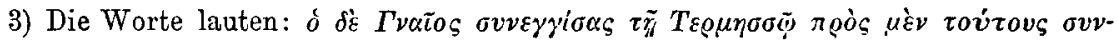

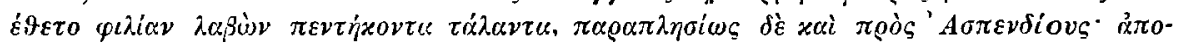

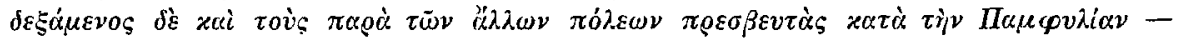

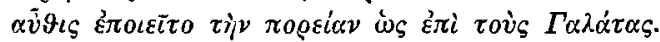


Nachricht über den oben erwähnten Streit des Eumenes und Antiochus über den Besitz Pamphyliens. Bei der Auslegung der beiden Stellen des Polybins und Livius ist Mommsen nümlich ein Versehen untergelaufen. Er stützt sich auf die Worte des Livius. die aber, wie manches andere in diesen Partien, infolge der Flüchtigkeit dieses Schriftstellers aus dem Polybius falsch übersetzt sind. Polybius sagt - man vergleiche den oben angeführten Wortlaut - Eumenes habe behauptet, Pamphylien liege diesseits, Antiochus dagegen, es liege jenseits des Taurus. Von einer Teilung von Pamphylien ist nicht die Rede, die hat erst Livius hineingebracht, indem er die

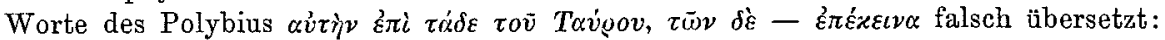
quia pars eius citra, pars ultra Taurum est. Aus Polybius ergibt sich mit Sicherheit, dass das Gebirge gemeint sein muss; ob Livius hier an den von ihm 38,15, 7 genannten Taurusfluss gedacht hat, ist zweifelhaft, möglich jedenfalls, weil er sonst Taveos mit Taurus mons zu übersetzen pflegt. Aber da uns das Original der Liviusstelle erhalten ist und dies keinen Zweifel der Auffassung zulässt, müssen wir Livius ganz beiseite lassen. Somit muss also definitivder Taurusflussals Grenze a us s h eide n, wie es ja denn überhaupt unwahrscheinlich ist, dass man einen Fluss, über dessen Identität Zweifel entstehen konnten, als Grenzscheide gewählt hïtte. Mommsen hat aber weiter nicht genug beachtet, - und damit komme ich zu seinem zweiten Einwand -, dass der Begrifi cis Taurum montem ein ganz feststehender ist.

Pol. 21, 14, 8 heisst es bei der ersten Verhandlung zwischen Scipio und Antio-

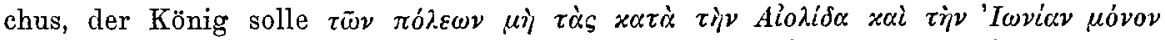

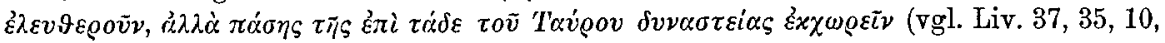
App. Syr. 29). Ebenso heisst es bei der zweiten Verhandlung zwischen den Krieg-

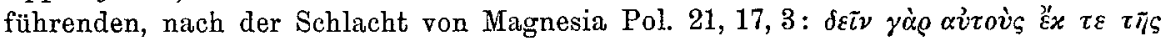

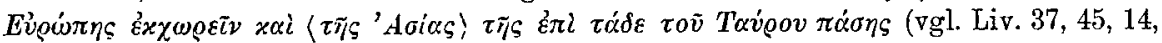

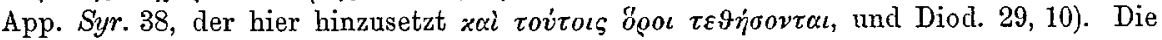
gleiche Bestimmung kehrt endlich wieder bei den Verhandlungen in Rom (vgl. Pol.

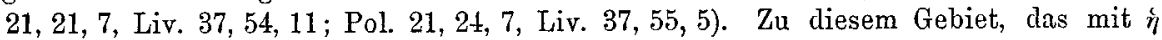
$\dot{\xi} \pi i \tau \dot{\alpha} \delta \varepsilon \quad \tau \circ \bar{v}$ Tav́oov bezeichnet wird, gehört, wie ausser den angeführten Stellen noch Pol. 21, 22, 14 (= Liv. 37, 54, 11), Liv. 37, 56, 2, Pol. 21, 48 ergeben, unter anderem auch Phrygien und Lykaonien, im ganzen genommen also die Landschaften Kleinasiens, die ausserhalb der vom Taurus eingeschlossenen Südküste Kleinasiens liegen, d. h. alles ausser Kilikien, Pisidien und ev. Pamphylien ${ }^{1}$ ). Diese Auffassung stimmt überein mit dem, was Strabo über den Taurus und über den Begriff $\tau \dot{\alpha}$ ćvtòs $\tau o \bar{v}$

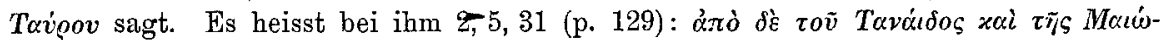

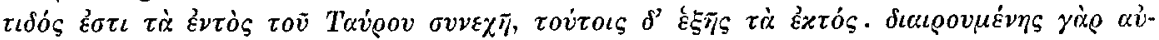

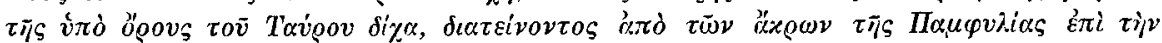

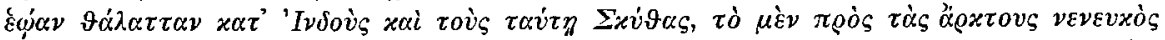

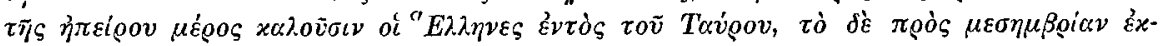
rós etc. (vgl. 11, 1, 2 p. $490 ; 11,12,1$ p. 520 etc.).

Aber auch der Einwand Mommsens ist hinfällig, den Taurus ohne nähere Definition als Grenze zu setzen, sei ein Nonsens. Denn wie verderbt auch die Worte des Livius sein mögen, so viel geht doch ans ihnen sicher hervor, dass mit ihnen irgendwie eine weitere Grenzlinie, die den Taurus berührte oder von ihm ausging, bezeichnet werden sollte, und diese Linie kann naturgemäss nur eine sein, die, im grossen und ganzen von Norden nach Süden laufend, den Machtbereich, die Interessensphäre der Römer von der des Antiochus schied. Dass bei Festsetzung dieser Linie nicht nur, wie Mommsen annehmen zu müssen glaubt, die Länder und Städte, die Antiochus wirklich besessen hatte, sondern auch ihm verbündete Landschaften wie Lykaonien, das noch dazu dem Reiche des Eumenes einverleibt wurde, mit eingeschlossen wurden, erscheint mir eigentlich so selbstverständlich, dass es nicht einer

1) Vgl. darüber weiter unten. 
besonderen Begründung bedarf. Da Kappadokien selbständig blieb, musste die Grenze zwischen Lykaonien und Kappadokien laufen, und damit kam man an den Halys, der schon von Crösus Zeit her als Grenzfluss zwischen dem westlichen und östlichen Kleinasien galt ${ }^{1}$ ). $\mathrm{Ob}$ das Gebiet der Galater bis zun Halys unterworfen war oder nicht, spielte für die Einsetzung des Halys in den Friedensvertrag keine Rolle. Für die Römer kam es darauf an, dass Antiochus alles Gebiet westlich von dieser Linie als ausserhalb seiner Interessensphäre liegend anerkannte. Doch will ich ausdrücklich darauf hinweisen, dass man an einen Feldzug gegen die Galater schon damals dachte, wie aus Livius 37, 51, 10 hervorgeht: metu, ne cum Gallis foret bellandum.

Auf Grund all dieser Erwägungen glaube ich die Worte des Livius so emendieren zu müssen: excedito urbibus agris vicis castellis cis Taurum montemusque ad Halyn amnem et ab eavalle (d. h. vom Tale des Halys aus, s. unten Anm. 1) usque ad i uga Tauri ${ }^{2}$ ), qua in L y c a oniam vergit, wobei m. E. mit diesen letzten Worten eine Linie bezeichnet werden soll, die von dem Punkt, wo der Halys sich nach Norden wendet, südlich etwa nach der Grenze von Kilikien und Pisidien verläuft, so dass also nach Meinung der Römer auch Lykaonien eingeschlossen war ${ }^{3}$ ). Nur Kilikien bleibt bei Antiochus, weil es

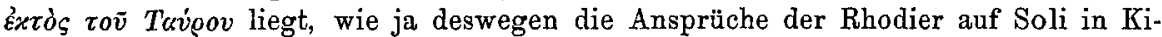
likien vom römischen Senat zurückgewiesen werden ${ }^{4}$ ). Pamphylien wurde von Eumenes und wohl auch von den Römern ${ }^{b}$ ) als diesseits des Taurus liegend in Anspruch genommen. Wenn Antiochus behauptete, es liege wie Kilikien jenseits des Taurus, so fasst er die Gebirgszüge, die Pamphylien halbmondförmig umschliessen

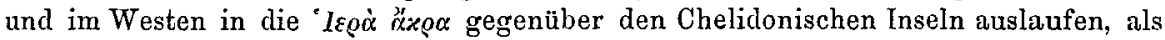
die westlichen Ausliiufer des Taurus, und zwar, wie aus Strabo hervorgeht ${ }^{6}$ ), in Uebereinstimmung mit der Mehrzahl der Geographen. Eumenes aber rechnete als Taurus

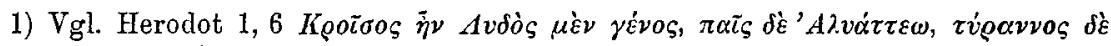

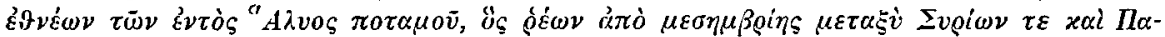

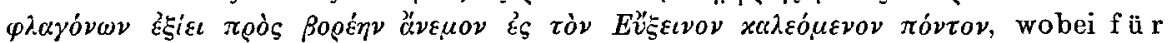
die Grenze auch nur der Mittel-und Unterlauf berücksichtigt wird. Siehe auch 1, 28. 72. 103.130 und Strab. 2, 5, 31 (p. 129) und 6, 4, 2 (p. 287) u. a.

2) Die Stellung usque ad iuga Tauri statt Tauri usque ad iuga scheint der Livianische Sprachgebrauch zu fordern.

3) Möglich ist auch, dass an eine Linie gedacht worden ist, die südwestlich verlief, so dass Lykaonien ausgeschlossen war (dies hat Müller akzeptiert), doch scheint mir wegen der oben vorgetragenen Erwïgungen die andere Ansicht den Vorzug zu verdienen.

4) Vgl. Pol. 21, 24, $10 \mathrm{ff} .$, Liv. 37, 56, $7 \mathrm{ff}$.

5) Dafür spricht, dass Antiochus nach dem Friedensvertrage mit seinen Schiffen nicht über das Sarpedonische Vorgebirge in Kilikien hinausfahren durfte (Pol. 21, 45, 14, Liv. 38, 38, 9, App. Syr. 39, vgl. Mommsen, Röm. Forsch. II S. 535), weiter das Eingreifen Volsos in die pamphylischen Angelegenheiten (vgl. oben S. 372 f.) und schliesslich Liv. 44, 14, 3, der unter d. J. 169 v. Chr. berichtet, Gesandte aus Pamphylien hätten einen goldenen Kranz überbracht und im Tempel des kapitolinischen Jupiter niedergelegt, worauf es weiter heisst: benigneque amicitiam renovare volentibus legatis responsum. Freilich scheint nach diesen Worten das Land für frei erklärt, nicht Eumenes zugesprochen worden zu sein.

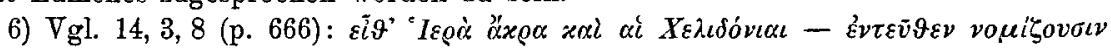

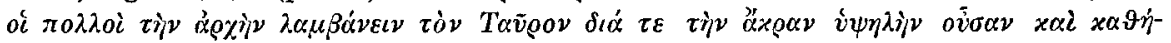

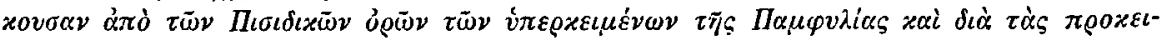

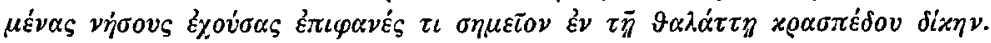


wohl nur den pisidischen und kilikischen, und dann konnte man freilich von Pamphylien ebenso wie von Lykien sagen, es liege diesseits des Taurus ${ }^{1}$ ).

Es bleibt nun noch die Frage übrig: Ist die Bestimmung, durch die der Halys im Osten als Grenze festgesetzt wird, schon 189 in den in Rom vom Senat abgeschlossenen und von den Parteien beschworenen, nach Appian Syr. 39 auf dem Kapitol aufgestellten Vertrag aufgenommen gewesen oder ist sie erst von der Zehnmännerkommission und Volso, der inzwischen ja bis an den Halys im Kampfe mit den Galatern vorgedrungen war, 188 bei der definitiven Ordnung in Apamea hinzugefügt worden? Ich habe an sich kein Bedenken, das erste anzunehmen, da ich ja

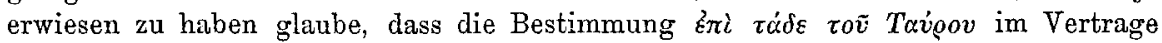
gestanden und diese zu ihrer Ergänzung die vom Taurus nach Norden gehende, Kleinasien in eine westliche und östliche Hälfte teilende Linie nötig hatte $\left.{ }^{2}\right)$. Es bleibt aber für den, der mit Mommsen und Müller eine solche Grenzbestimmung im Jahre 189 in Rom für nicht glaublich hält, die Möglichkeit, anzunehmen, dass der Zusatz erst in Apamen gemacht worden ist. Dann muss entweder Polybius den erweiterten Text von Apamea benutzt haben oder die Grenzbestimmung nachtrïglich dem in Rom aufgestellten Bündnisvertrag hinzugefügt worden sein ${ }^{3}$ ). Dass Volso und die Zehnmännerkommission berechtigt waren, solche Zusïtze zu machen, unterliegt keinem

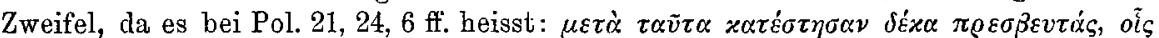

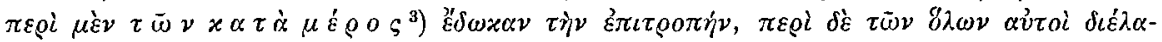

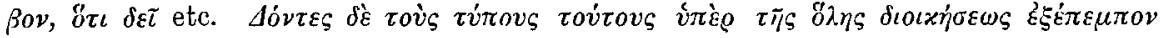

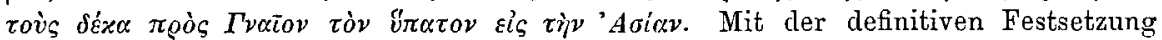
aller Einzelheiten hängt es auch zusammen, dass der Vertrag, obwohl schon in Rom beschworen (Pol. 21, 24,3), doch von neuem in Apamea (ibid. c. 44, 10) und dann sogar noch einmal in Syrien von Antiochus selbst beschworen wurde, zu dem, wie

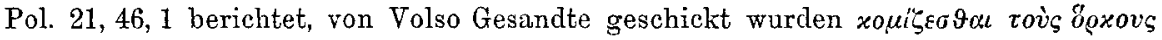

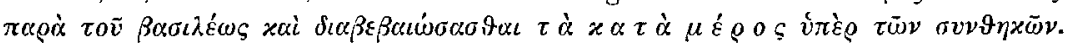

Berlin.

Zur Verwaltungsgeschichte der römischen Eisenbergwerke in Dakien.

Die dakischen Eisenwerke im Gebiete von Apulum (Karlsburg-Gyulafehérvar) befanden sich im zweiten Jahrhundert nach Ausweis einer im Jahre 1840 von Regimentspater Daniel Thalson dort gefundenen Inschrift (CIL. III 1128), wie schon Mommsen gesehen hat, in kâserlichem Besitz, und zwar hat aller Wahrscheinlichkeit nach wie in Gallien unter dem Provinzialprokurator ein besonderer Bergwerksprokurator (CIL. VI 31863 procurator Aug. ferraricurum) oder wenigstens ein villicus gestanden.

In der Nähe von Vajdahunyad (Hunya der Comitat) bei Alsó Telek $10 \mathrm{~km}$ östlich von Gyalír, wo die dakischen Eisenerzlager ihren Mittelpunkt hatten, glückte es mir im Jahre 1883 tatsïchliche Beweise der römischen Bergwerksarbeit in Gestalt von Werkzengen zu entdecken, die jetzt im Dewaer Museum aufbewahrt sind. Einen näheren Einblick in die innere Organisation der dakischen Eisenwerke

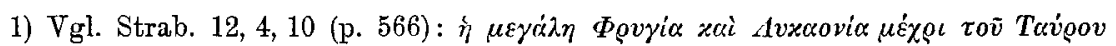

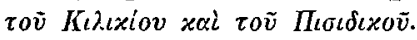

2) Damit würde stimmen, dass App. Syr. 38 den Scipio bei der-Forderung der

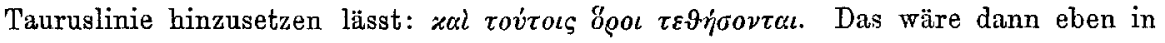
Rom geschehen.

3) Vgl. z. B. das Senatskonsult von Thisbae (Viereck, Sermo graecus p. 12 ff.), in dem die Erledigung verschiedener Sachen dem Praetor Q. Maenius und dem Konsu]

A. Hostilius übertragen wird. Siehe auch die vorige Anm.

Klio, Beitrüge zur alten Gegchichte IX 3. 